\title{
Representations with regular norm-behaviour of locally compact abelian semigroups
}

by

\author{
LÁsZló KÉRCHY and Zoltán LÉKA (Szeged)
}

\begin{abstract}
We prove that some regularity conditions on unbounded representations of topological abelian semigroups with countable spectral conditions induce a certain stability result extending the well-known Arendt-Batty-Lyubich-Vũ theorem.
\end{abstract}

1. Introduction. Consider the well-posed abstract Cauchy problem

$$
(\mathrm{ACP}) \quad\left\{\begin{array}{l}
\dot{u}(t)=A u(t) \quad(t \geq 0), \\
u(0)=x
\end{array}\right.
$$

where $A$ is a densely defined, closed operator acting on a complex Banach space $\mathcal{X}$ and $x \in \mathcal{X}$. Let $(T(t))_{t \geq 0}$ denote the $\mathrm{C}_{0}$-semigroup generated by $A$ and suppose that the orbits $T(t) x$ are bounded for all $x \in \mathcal{X}$. The well-known Arendt-Batty-Lyubich-Vũ theorem asserts that $\lim _{t \rightarrow \infty}\|T(t) x\|=0$ for all $x \in \mathcal{X}$ if $\sigma(A) \cap i \mathbb{R}$ is countable and the point spectrum of the adjoint $A^{*}$ on $i \mathbb{R}$ is empty. Quite a few generalizations of the theorem are known for bounded and unbounded representations of suitable locally compact abelian semigroups (see $[\mathrm{B}],[\mathrm{BV}],[\mathrm{BY}],[\mathrm{K} 1],[\mathrm{K} 2])$. In this article we shall extend to topological semigroups the method that appears in the papers [K1], [K2] in connection with discrete abelian semigroups.

In the next section we introduce the concept of almost convergence (defined for sequences in [Lo]) in terms of invariant means, which will be crucial in defining the limit functional in Section 3 for unbounded representations satisfying a certain regularity condition. It turns out that this limit functional is intrinsic to the representation itself and independent of any choice of gauge function involved in the regularity condition. This enables us to define the peripheral spectrum, and various spectral notions of representations with regular norm-function, in Section 4. In the bounded case the definition

2000 Mathematics Subject Classification: Primary 47D03.

Key words and phrases: representations of semigroups, regular norm-function, almost convergence, peripheral spectrum.

Research partially supported by Hungarian NSRF (OTKA) grant no. T 49846. 
coincides with the one introduced by Batty and Vũ [BV] and Lyubich [L]. In Section 5 we generalize a well-known method associating an isometric representation to a representation with regular norm-function. With these results at hand, we give a new extension of the ABLV theorem in Section 6. Our stability theorem is strongly related to the main result of [BY]. The spectral conditions are essentially the same. The differences appear in the method of normalization and in the nature of convergence expressing stability. Our proof follows the usual scheme and relies on results about isometric representations proved in [BV]. The originality of our approach is in the usage of almost convergence defining regular norm-behaviour and in the systematic study of representations with this property. We note that $[\mathrm{V}]$ was the first paper dealing with a weighted version of the ABLV theorem. The more recent work $[\mathrm{BP}]$ has a very general result, in the spirit of $[\mathrm{BY}]$, but using different methods.

2. Almost convergence on semigroups. Consider an abelian semigroup $(S,+)$. For any $s \in S$ and $\omega \subseteq S$ set $\omega \ominus s:=\left\{s^{\prime} \in S: s+s^{\prime} \in \omega\right\}$. The translation of a function $f: S \rightarrow \mathbb{C}$ by $s \in S$ is the mapping $f_{s}: S \rightarrow \mathbb{C}$ defined by $f_{s}\left(s^{\prime}\right):=f\left(s+s^{\prime}\right) \quad\left(s^{\prime} \in S\right)$. Let $(S, \Omega, \lambda)$ be a $\sigma$-finite measure space on $S$ such that (i) $\omega \ominus s \in \Omega$ whenever $\omega \in \Omega$ and $s \in S$, and (ii) $\lambda(\omega)=0(\omega \in \Omega)$ implies $\lambda(\omega \ominus s)=0$ for all $s \in S$. Obviously, $S=\mathbb{R}_{+}^{n}$ with the Lebesgue measure and $S=\mathbb{Z}_{+}^{n}$ with the counting measure satisfy these conditions. Let $L^{\infty}(\lambda)$ denote the Banach space of all essentially bounded, complex-valued, measurable functions with the usual norm.

LEMma 1. Under the conditions (i) and (ii), $\tau_{s}: L^{\infty}(\lambda) \rightarrow L^{\infty}(\lambda), f \mapsto f_{s}$, is a well-defined linear mapping with $\left\|\tau_{s}\right\|=\left\|\tau_{s} \mathbf{1}\right\|=1$ for every $s \in S$.

Proof. Let $\chi_{\omega}$ be the characteristic function of $\omega \in \Omega$. Since $\chi_{\omega}$ is measurable we infer by (i) that $\left(\chi_{\omega}\right)_{s}=\chi_{\omega \ominus s}$ is also measurable. Using the usual approximation method we find that $f_{s}$ is measurable for any $f \in L^{\infty}(\lambda)$. To see that the translation is well-defined on $L^{\infty}(\lambda)$ suppose that $f_{1}=f_{2}$ a.e.; then by (ii) we have $\left(f_{1}\right)_{s}=\left(f_{2}\right)_{s}$ a.e. Obviously, $\left\|f_{s}\right\|_{\infty} \leq\|f\|_{\infty}$. Since $\|\mathbf{1}\|_{\infty}=1$ and $\tau_{s} \mathbf{1}=\mathbf{1}$, we obtain $\left\|\tau_{s}\right\|=\left\|\tau_{s} \mathbf{1}\right\|=1$.

The set $\widetilde{M}(S, \lambda):=\left\{m \in L^{\infty}(\lambda)^{*}:\|m\|=m(\mathbf{1})=1\right\}$ is called the set of means on $S$ with respect to $\lambda$. The Hahn-Banach theorem implies that $\widetilde{M}(S, \lambda) \neq \emptyset$. A functional $m \in \widetilde{M}(S, \lambda)$ is called an invariant mean if $m\left(f_{s}\right)=m(f)$ for every $f \in L^{\infty}(\lambda)$ and $s \in S$; the set of all invariant means with respect to $\lambda$ is denoted by $M(S, \lambda)$. It is well-known that there are invariant means on abelian groups, and this can be easily extended to semigroups. For the reader's convenience we sketch the proof.

Proposition 2. $M(S, \lambda)$ is not empty. 
Proof. Since $\widetilde{M}(S, \lambda)$ is a convex, weak-* closed subset of the unit ball of $L^{\infty}(\lambda)^{*}$, it is weak-* compact. Taking an $m \in \widetilde{M}(S, \lambda)$, for any $s \in S$ we have $m \circ \tau_{s} \in \widetilde{M}(S, \lambda)$, and $T(s): \widetilde{M}(S, \lambda) \rightarrow \widetilde{M}(S, \lambda), m \mapsto m \circ \tau_{s}$, is an affine, weak-* continuous mapping. The Markov-Kakutani theorem (see e.g. [C, Theorem V.10.1]) implies the existence of a common fixed point $m_{0} \in \widetilde{M}(S, \lambda)$. It is immediate that $m_{0}$ is an invariant mean.

Consider a locally compact, Hausdorff abelian group $G$. Let $S$ be a closed subsemigroup of $G$ with nonempty interior $S^{\circ}$ such that $S-S=G$ and $S \cap(-S)=\{0\}$. By definition, for any $s_{1}, s_{2} \in S, s_{1} \preceq s_{2}$ if $s_{2}-s_{1} \in S$. In this way we obtain an inductive partial ordering on $S$, hence $S$ is a directed set. We say that a function $f: S \rightarrow \mathbb{C}$ tends to 0 at infinity, written $\lim _{s} f(s)=0$, if for every $\varepsilon>0$ there exists an $s_{0} \in S$ such that $|f(s)|<\varepsilon$ whenever $s_{0} \preceq s$. Let $\mu$ denote the restriction of the Haar measure $\widetilde{\mu}$ of $G$ to $S$. It is clear that conditions (i) and (ii) of Lemma 1 are satisfied for $\mu$, indeed: $\omega \ominus s=(\omega-s) \cap S$. The (invariant) means with respect to $\mu$ are simply called (invariant) means on $S$, and the notation $L^{\infty}(S):=L^{\infty}(\mu), \widetilde{M}(S):=$ $\widetilde{M}(S, \mu), M(S):=M(S, \mu)$ is used. Throughout the paper a function $f$ defined on $S$ is also considered to be a function on $G$, zero outside $S$.

Definition. A net $\left\{K_{\lambda}\right\}_{\lambda \in \Lambda}$ of compact subsets of $G$ with nonempty interiors is a strong Følner net for $G$ if

(i) $K_{\lambda_{1}} \subseteq K_{\lambda_{2}}$ whenever $\lambda_{1} \leq \lambda_{2}$,

(ii) $G=\bigcup K_{\lambda}^{\circ}$,

(iii) $\widetilde{\mu}\left(\left(x+K_{\lambda}\right) \triangle K_{\lambda}\right) / \widetilde{\mu}\left(K_{\lambda}\right) \rightarrow 0$ (as $\lambda \rightarrow \infty$ ) uniformly when $x$ is running through compact sets. (Here and below, $\triangle$ stands for the symmetric difference.)

A net $\left\{K_{\lambda}\right\}_{\lambda \in \Lambda}$ of compact subsets of $G$ with nonempty interiors is called a Følner net for $G$ if (iii) is satisfied.

For example, if $G=\mathbb{R}$ then $K_{n}:=[-n, n]$ is a Følner sequence. We recall that an arbitrary locally compact group $G$ is called amenable if there exists an invariant mean on $G$. The following theorem is a useful and deep characterization of amenable groups (for its proof we refer to $[\mathrm{P}$, Theorem $4.16])$.

TheOREM 3. A locally compact group $G$ is amenable if and only if there exists a strong Følner net for $G$. If $G$ is $\sigma$-compact, then $G$ is amenable if and only if there exists a strong Følner sequence for $G$.

We shall assume in the following that $G$ is $\sigma$-compact. Since $G$ is abelian by our assumption, the Markov-Kakutani theorem implies that $G$ is amenable (see the proof of Proposition 2), thus using the characterization theorem we obtain the existence of a strong Følner sequence $\left\{K_{n}\right\}_{n \in \mathbb{N}}$ on $G$. We 
shall translate this sequence to the interior of $S$, preserving property (iii), by means of the following two lemmas.

Lemma 4. If $\left\{K_{n}\right\}_{n}$ is a Følner sequence for $G$ then $\left\{K_{n}+s_{n}\right\}_{n}$ is also a Følner sequence for $G$, for every sequence $\left\{s_{n}\right\}_{n=1}^{\infty}$ in $G$.

Proof. Using the translation invariance of the Haar measure, for any measurable sets $B_{1}, B_{2}$ and $g \in G$, we have $\widetilde{\mu}\left(\left(B_{1}+g\right) \triangle\left(B_{2}+g\right)\right)=$ $\widetilde{\mu}\left(B_{1} \triangle B_{2}\right)$; hence $\left\{K_{n}+s_{n}\right\}_{n}$ is also a Følner sequence.

Lemma 5. Let $K$ be a compact set in $G$. Then there exists an $s \in S$ such that $K+s \subseteq S^{\circ}$.

Proof. Since $G=S-S$ by assumption, for any $g \in G$ and $s_{0} \in S^{\circ}$ we have $g-s_{0}=s^{\prime}-s^{\prime \prime}$ with some $s^{\prime}, s^{\prime \prime} \in S$. Since clearly $s_{0}+s^{\prime} \in S^{\circ}$, it follows that $G=S^{\circ}-S$. The family $\left\{S^{\circ}-s: s \in S\right\}$ forms an open covering of $K$ and so there exist $s_{1}, \ldots, s_{n} \in S$ such that $K \subseteq \bigcup_{i=1}^{n}\left(S^{\circ}-s_{i}\right)$. Set $s=\sum_{i=1}^{n} s_{i}$. As $s_{i} \preceq s$, we have $S^{\circ}-s_{i} \subseteq S^{\circ}-s$ for every $1 \leq i \leq n$. It follows that $K \subseteq S^{\circ}-s$, that is, $K+s \subseteq S^{\circ}$.

An immediate consequence of Lemmas 4 and 5 is that there is a Følner sequence in the interior of $S$.

We shall need yet the following auxiliary result on topological semigroups. Recall that a function $f$ on $S$ is called locally bounded if it is bounded on compact subsets of $S$. Let $C_{\mathrm{c}}(S)$ denote the set of continuous functions with compact support in $S$.

Lemma 6. Let $K \subseteq S^{\circ}$ be a compact subset of $G$. If $f$ is a measurable, locally bounded function on $S$ and $g \in L^{\infty}(S)$ then the convolution

$$
(f * g)(s):=\int_{K} f(s+t) g(t) d \mu(t)
$$

is continuous on $S$.

Proof. Assuming $f \in C_{\mathrm{c}}(S)$, we can easily verify that $f$ is uniformly continuous on $S$, that is, for every $\varepsilon>0$, there exists an open set $U$ in $G$ containing 0 such that $\left|f\left(s^{\prime}\right)-f(s)\right| \leq \varepsilon$ whenever $s^{\prime}-s \in U$ and $s^{\prime}, s \in S$. Hence $f * g$ is continuous.

Suppose now that $f$ is any locally bounded, measurable function. We can choose a compact neighbourhood $K_{1}$ of 0 in $G$ such that $K+K_{1} \subseteq S^{\circ}$. Indeed, every $s \in K$ is an inner point of $S$, hence there exists an open neighbourhood $V_{s}$ of 0 (in $G$ ) such that $s+V_{s} \subseteq S^{\circ}$. Choose open neighbourhoods $W_{s}$ of 0 such that $W_{s}+W_{s} \subseteq V_{s}$. Then $\left\{s+W_{s}: s \in K\right\}$ is an open covering of $K$ and therefore has a finite subcovering $s_{1}+W_{s_{1}}, \ldots, s_{n}+W_{s_{n}}$. The set $W:=\bigcap_{i=1}^{n} W_{s_{i}}$ is open and $K+W \subseteq\left(\bigcup_{i=1}^{n}\left(s_{i}+W_{s_{i}}\right)\right)+W=$ $\bigcup_{i=1}^{n}\left(s_{i}+W_{s_{i}}+W\right) \subseteq \bigcup_{i=1}^{n}\left(s_{i}+V_{s_{i}}\right) \subseteq S^{\circ}$. Since $G$ is a locally compact 
Hausdorff space there is a compact neighbourhood $K_{1} \subseteq W$ of 0 , hence $K_{2}:=K+K_{1} \subseteq S^{\circ}$. It is clear that $K_{2}$ is also compact.

Fix $\varepsilon>0$ and $y \in S$. Since $f \in L^{1}\left(S, \chi_{K_{2}+y} d \mu\right)$, there exists an $h \in C_{\mathrm{c}}(S)$ such that $\int_{K_{2}+y}|f(t)-h(t)| d \mu(t)<\varepsilon$. (Notice that the restricted Haar measure $\mu$ is regular on the $\sigma$-compact set $S$; see [Pe, p. 238].) We already know that $h * g$ is continuous. Hence we can choose a neighbourhood $U$ of 0 in $G$ such that $U \subseteq K_{1}$ and $|(h * g)(y)-(h * g)(v)|<\varepsilon$ whenever $v \in(y+U) \cap S$. Then

$$
\begin{aligned}
|(f * g)(y)-(f * g)(v)| \leq & \int_{K}\left|f_{y}-h_{y}\right||g| d \mu+|(h * g)(y)-(h * g)(v)| \\
& +\int_{K}\left|h_{v}-f_{v}\right||g| d \mu \\
< & \int_{K+y}|f-h|\|g\|_{\infty} d \mu+\varepsilon+\int_{K+v}|h-f|\|g\|_{\infty} d \mu \\
\leq & 2\|g\|_{\infty} \int_{y+K_{2}}|f-h| d \mu+\varepsilon<2 \varepsilon\|g\|_{\infty}+\varepsilon,
\end{aligned}
$$

and the lemma follows.

We proceed to the study of almost convergence.

Definition. A function $f \in L^{\infty}(S)$ is called almost convergent if the set $\{m(f): m \in M(S)\}$ is a singleton. We shall use the notation a-lim $f=c$ whenever $m(f)=c$ for all $m \in M(S)$.

A useful property of almost convergence is described in the following proposition.

Proposition 7. If $f \in L^{\infty}(S)$ is almost convergent with a-lim $f=c$ and $\left\{K_{n}\right\}_{n}$ is a Følner sequence on $S$ then

$$
\lim _{n \rightarrow \infty} \frac{1}{\mu\left(K_{n}\right)} \int_{K_{n}} f_{y} d \mu=c
$$

uniformly with respect to $y \in S$.

Proof. Assume that there exist an $\varepsilon>0$, a strictly increasing sequence $\left\{n_{k}\right\}_{k}$ of positive integers, and a sequence $\left\{y_{k}\right\}_{k} \subset S$ such that

$$
\left|\frac{1}{\mu\left(K_{n_{k}}\right)} \int_{K_{n_{k}}} f_{y_{k}} d \mu-c\right|>\varepsilon \quad \text { for every } k \in \mathbb{N} .
$$

Applying Lemma 4 to the subsequence $\left\{K_{n_{k}}\right\}_{k}$ we see that $\left\{C_{k}:=K_{n_{k}}+y_{n_{k}}\right\}_{k}$ is also a Følner sequence. For every $k \in \mathbb{N}$, consider the linear functional $\varphi_{k}$ 
on $L^{\infty}(S)$ defined by

$$
\varphi_{k}(g):=\frac{1}{\mu\left(C_{k}\right)} \int_{C_{k}} g d \mu, \quad g \in L^{\infty}(S) .
$$

Obviously, $\left\|\varphi_{k}\right\|=\varphi_{k}(\mathbf{1})=1$. By the Banach-Alaoglu theorem there exists a weak-* cluster point $m_{0}$ of $\left\{\varphi_{k}\right\}_{k}$. Clearly, $\left\|m_{0}\right\|=m_{0}(\mathbf{1})=1$. Furthermore, for every $g \in L^{\infty}(S)$ and $y \in S$, we have

$$
\begin{aligned}
\left|\varphi_{k}(g)-\varphi_{k}\left(g_{y}\right)\right| & =\frac{1}{\mu\left(C_{k}\right)}\left|\int_{C_{k}} g d \mu-\int_{C_{k}+y} g d \mu\right| \\
& \leq \frac{\mu\left(\left(C_{k}+y\right) \triangle C_{k}\right)}{\mu\left(C_{k}\right)}\|g\|_{\infty} \rightarrow 0 \quad \text { as } k \rightarrow \infty,
\end{aligned}
$$

whence $m_{0}(g)=m_{0}\left(g_{y}\right)$, that is, $m_{0}$ is an invariant mean.

Since $\left|m_{0}(f)-c\right| \geq \varepsilon$ by our assumption, we conclude that $f$ cannot almost converge to $c$.

We shall also need a stronger form of almost convergence.

Definition. We say that a function $f \in L^{\infty}(S)$ almost converges in strong sense to a complex number $c$ if a-lim $|f-c|=0$. In that case we shall write as-lim $f=c$.

The following multiplicative property of as-lim is analogous to [K2, Lemma 1]:

Lemma 8. For every $f \in L^{\infty}(S)$, the following statements are equivalent:

(i) $\operatorname{as}-\lim f=c$,

(ii) $m(f g)=c m(g)$ for every $g \in L^{\infty}(S)$ and $m \in M(S)$.

Proof. (i) $\Rightarrow$ (ii): Every invariant mean $m \in M(S)$ is a positive functional on $L^{\infty}(S)$. Given any $h \in L^{\infty}(S)$, let $\alpha$ be a complex number with $|\alpha|=1$ and $|m(h)|=\alpha m(h)$. Since $|h|-\operatorname{Re}(\alpha h) \geq 0$, it follows that $m(|h|) \geq$ $m(\operatorname{Re}(\alpha h))=\operatorname{Re} m(\alpha h)=|m(h)|$. Therefore

$$
|m(f g)-c m(g)|=|m(f g-c g)| \leq m(|f g-c g|) \leq\|g\|_{\infty} m(|f-c \mathbf{1}|)=0 .
$$

(ii) $\Rightarrow\left(\right.$ i): Let $g(s):=|f(s)-c|(f(s)-c)^{-1}$ if $f(s) \neq c$ and 0 otherwise. Then $g \in L^{\infty}(S)$, and $m(|f-c \mathbf{1}|)=m((f-c) g)=m(f g)-c m(g)=0$.

It is easy to check that convergence implies almost convergence in strong sense. Consider the function $f \in L^{\infty}\left(\mathbb{R}_{+}\right)$, where $f(s):=1$ if $n \leq s<n+1$ and $n$ is even, while $f(s):=-1$ otherwise. Since $f+f_{1}$ is identically zero, we infer that a- $\lim f=0$; however, $\lim _{s \rightarrow \infty} f(s)$ does not exist. It is also clear that $f$ does not converge in the strong sense. Consider now the function $g \in L^{\infty}\left(\mathbb{R}_{+}\right)$, where $g(s):=1$ if $2^{n} \leq s<2^{n}+1(n \in \mathbb{N})$, while $g(s):=0$ otherwise. For any $a \in \mathbb{R}_{+}$and $N \in \mathbb{N}$, let $G_{a, N}:=N^{-1} \sum_{k=1}^{N} g_{a+k}$. Since 
$m\left(G_{a, N}\right)=m(g)$ for every invariant mean $m \in M\left(\mathbb{R}_{+}\right)$, and since $\left\|G_{a, N}\right\|_{\infty}$ can be arbitrarily small, we conclude that as-lim $g=0$. On the other hand, $\lim _{s \rightarrow \infty} g(s)$ does not exist.

3. Existence of the limit functional. The regular norm-behaviour of representations will be defined in terms of gauge functions.

Definition. We say that $p: S \rightarrow(0, \infty)$ is a gauge function if it is locally bounded, measurable and, for every $s \in S, p_{s} / p \in L^{\infty}(S)$ almost converges in strong sense to a positive real number $c_{p}(s)$. The function $c_{p}$ is called the limit functional of the gauge function $p$.

If $p: S \rightarrow(0, \infty)$ is a gauge function then the equality

$$
\frac{p_{s_{1}+s_{2}}}{p}=\frac{\left(p_{s_{1}}\right)_{s_{2}}}{p_{s_{2}}} \cdot \frac{p_{s_{2}}}{p}
$$

implies by Lemma 8 that $c_{p}\left(s_{1}+s_{2}\right)=c_{p}\left(s_{1}\right) c_{p}\left(s_{2}\right)\left(s_{1}, s_{2} \in S\right)$, that is, the limit functional $c_{p}$ of $p$ is multiplicative.

The next lemma plays an important role in the proof of the main theorem of this section.

Lemma 9. Let $p$ be a gauge function with $p(s) \geq 1$ for $s \in S$. Then $c_{p}(s) \geq 1$ for every $s \in S$.

Proof. We can see from Proposition 7 that, for every $s \in S$,

$$
\frac{1}{\mu\left(K_{n}\right)} \int_{K_{n}}\left(p_{s} / p\right)_{y} d \mu \rightarrow c_{p}(s) \quad(n \rightarrow \infty)
$$

uniformly with respect to $y$, where $\left\{K_{n}\right\}_{n}$ is a Følner sequence in $S$. Assume that $c_{p}\left(s_{0}\right)<1$ for some $s_{0} \in S$, and choose $c_{p}\left(s_{0}\right)<\delta<1$. There exists $n_{0} \in \mathbb{N}$ such that $\mu\left(K_{n_{0}}\right)^{-1} \int_{K_{n_{0}}}\left(p_{s_{0}} / p\right)_{y} d \mu \leq \delta$ is true for every $y \in S$. Now the Jensen inequality implies

$$
\begin{aligned}
\frac{1}{\mu\left(K_{n_{0}}\right)} \int_{K_{n_{0}}}\left(\log p_{s_{0}+y}-\log p_{y}\right) d \mu & \leq \log \left(\frac{1}{\mu\left(K_{n_{0}}\right)} \int_{K_{n_{0}}}\left(p_{s_{0}} / p\right)_{y} d \mu\right) \\
& \leq \log \delta<0 \quad(y \in S) .
\end{aligned}
$$

Summing the inequalities obtained for $y=0, s_{0}, 2 s_{0}, \ldots,(m-1) s_{0}$ we deduce that

$$
\frac{1}{\mu\left(K_{n_{0}}\right)} \int_{K_{n_{0}}} \log p_{m s_{0}} d \mu \leq \frac{1}{\mu\left(K_{n_{0}}\right)} \int_{K_{n_{0}}} \log p d \mu+m \log \delta
$$

for every $m \in \mathbb{N}$. This is a contradiction because the left-hand side of the inequality must be nonnegative, since $p \geq 1$, but the right-hand side is negative for large $m$. 
We recall that the continuous homomorphisms of $S$ into the multiplicative semigroup of $\mathbb{C}$, not identically zero, are called the characters of $S$.

COROLlary 10. Let $\chi$ be a character of $S$ such that $c_{p} \leq|\chi| \leq p$. Then $|\chi|=c_{p}$.

Proof. It is clear that $\widetilde{p}=p /|\chi|$ is a gauge function with $c_{\widetilde{p}}=c_{p} /|\chi|$. Since $\widetilde{p} \geq 1$, Lemma 9 yields $c_{\widetilde{p}} \geq 1$, and so $c_{p} \geq|\chi|$.

Throughout the paper $\mathcal{X}$ stands for a complex Banach space and $\mathcal{L}(\mathcal{X})$ is the set of all bounded linear operators on $\mathcal{X}$. A representation $\varrho$ of $S$ in the Banach space $\mathcal{X}$ is a mapping $\varrho: S \rightarrow \mathcal{L}(\mathcal{X})$ such that $\varrho(0)=I, \varrho(s+t)=$ $\varrho(s) \varrho(t)(s, t \in S)$, and $s \mapsto \varrho(s) x$ is continuous for every $x \in \mathcal{X}$.

Proposition 11. Let $\varrho: S \rightarrow \mathcal{L}(\mathcal{X})$ be a bounded representation. Then, for every $x \in \mathcal{X},\|\varrho(\cdot) x\|$ almost converges to zero if and only if $\lim _{s}\|\varrho(s) x\|$ $=0$.

Proof. Assume a- $\lim _{s}\|\varrho(s) x\|=0$ for some $x \in \mathcal{X}$. Then by Proposition 7 , for any $\varepsilon>0$, we have $\left\|\varrho\left(s_{0}\right) x\right\| \leq \varepsilon M^{-1}$ for some $s_{0} \in S$, where $M:=\sup \{\|\varrho(s)\|: s \in S\}$. Thus $\left\|\varrho\left(s+s_{0}\right) x\right\| \leq M \varepsilon M^{-1}=\varepsilon$ for every $s \in S$, and so $\lim _{s}\|\varrho(s) x\|=0$. The converse is trivial.

Now we introduce the concept of regularity.

Definition. The representation $\varrho: S \rightarrow \mathcal{L}(\mathcal{X})$ is of regular normbehaviour with respect to the gauge function $p$ or has $p$-regular norm-function if $\|\varrho(s)\| \leq p(s)$ for every $s \in S$, and a- $\lim _{s}\|\varrho(s)\| / p(s)=0$ does not hold.

There is a connection between the spectral radius function $r(\varrho(s))$ of a representation $\varrho$ with $p$-regular norm-function and the limit functional $c_{p}(s)$. Namely, the argument applied in the proof of [K2, Proposition 8] yields the following statement:

Lemma 12. $c_{p}(s) \leq r(\varrho(s))$ for every $s \in S$.

We are basically interested in unbounded representations of $S$, and the gauge functions will be used to derive bounded functions. Therefore the following assumption, valid throughout the paper, is natural: for any gauge function $p$ we have $p \geq 1$.

We show that the limit functional $c_{p}$ is continuous.

THEOREM 13. Let $p$ be a gauge function on $S$ satisfying $(\mathrm{H})$ and assume that there exists a representation $\varrho: S \rightarrow \mathcal{L}(\mathcal{X})$ with a p-regular normfunction. Then the limit functional $c_{p}$ of $p$ is a positive character of $S$.

Proof. We already know that $c_{p}\left(s^{\prime}+s^{\prime \prime}\right)=c_{p}\left(s^{\prime}\right) c_{p}\left(s^{\prime \prime}\right), s^{\prime}, s^{\prime \prime} \in S$, so $c_{p}$ is a homomorphism. It remains to show that $c_{p}$ is continuous. First, in 
view of Proposition 7 and Lemma 6, we see that $c_{p}$ is a measurable function, being a sequential limit of continuous functions.

The Uniform Boundedness Principle shows that the function $\|\varrho(\cdot)\|$ is bounded on compact sets, thus Lemma 12 implies that $c_{p}$ is also locally bounded on $S$. Choose a compact set $K \subseteq S^{\circ}$ such that $\mu(K)>0$. Then $\alpha:=\int_{K} c_{p}(t) d \mu(t) \in(0, \infty)$. Given any $y \in S$, we have

$$
\int_{K} c_{p}(y+t) d \mu(t)=\int_{K} c_{p}(y) c_{p}(t) d \mu(t)=c_{p}(y) \int_{K} c_{p}(t) d \mu(t),
$$

whence $c_{p}(y)=\alpha^{-1} \int_{K} c_{p}(y+t) d \mu(t)$. Now we can apply Lemma 6 to deduce the continuity of $c_{p}$.

Now we have arrived at the main result of the section.

THEOREM 14. Let $G$ be a locally compact, $\sigma$-compact, Hausdorff abelian group with a closed subsemigroup $S$ such that $S-S=G, S \cap(-S)=\{0\}$ and $S^{\circ} \neq \emptyset$. If the representation $\varrho: S \rightarrow \mathcal{L}(\mathcal{X})$ is of regular norm-behaviour with respect to the gauge functions $p$ and $q$ satisfying $(\mathrm{H})$, then

$$
c_{p}=c_{q} .
$$

Proof. We see from Lemma 12 that

$$
c_{p}(s) \leq r(\varrho(s)) \leq\|\varrho(s)\| \leq q(s) \quad(s \in S),
$$

and $c_{p}^{-1} q$ is a gauge function by Theorem 13 . Thus by Lemma 9 we have

$$
1 \leq c_{c_{p}^{-1} q}=c_{p}^{-1} c_{q}
$$

so $c_{p} \leq c_{q}$. In a similar way we find that $c_{p} \geq c_{q}$, thus $c_{p}=c_{q}$.

Now the following definition makes sense:

Definition. The function $c_{\varrho}:=c_{p}$ is called the limit functional of the representation @ with p-regular norm-function.

It was already shown in [K1] that for $S=\mathbb{Z}_{+}$the limit functional $c_{\varrho}(n)$ is equal to $r(\varrho(n))\left(n \in \mathbb{Z}_{+}\right)$. The analogous statement concerning $\mathrm{C}_{0}$-semigroups is also valid. To prove this, we need a lemma. We say that a representation $T: \mathbb{R}_{+} \rightarrow \mathcal{L}(\mathcal{X})$ is quasinilpotent if $r(T(s))=0$ for every $s>0$. (Notice that in the quasinilpotent case $r(T(\cdot))$ is not continuous, since $r(T(0))=1$, and so it is not a character.)

Lemma 15 . If the representation $T: \mathbb{R}_{+} \rightarrow \mathcal{L}(\mathcal{X})$ is not quasinilpotent then $r(T(\cdot))$ is a character of $\mathbb{R}_{+}$.

Proof. Since this statement is certainly well-known, we only sketch a proof. The submultiplicativity $r(T(s+t)) \leq r(T(s)) r(T(t))\left(s, t \in \mathbb{R}_{+}\right)$and $r(T(n s))=r(T(s))^{n}\left(n \in \mathbb{N}, s \in \mathbb{R}_{+}\right)$imply that $r(T(s))>0$ for every $s \in \mathbb{R}_{+}$. Furthermore, it can be easily checked that $\omega_{0}:=\lim _{s \rightarrow \infty} s^{-1} \log \|T(s)\|$ 
$\in \mathbb{R}$ exists; see e.g. [EN, p. 251]. Thus $r(T(s))=\lim _{n \rightarrow \infty}\|T(n s)\|^{1 / n}=$ $\exp \left(s \lim _{n \rightarrow \infty}(n s)^{-1} \log \|T(n s)\|\right)=\exp \left(\omega_{0} s\right)$ for every $s \in \mathbb{R}_{+}$.

Proposition 16. If the representation $T: \mathbb{R}_{+} \rightarrow \mathcal{L}(\mathcal{X})$ is of regular norm-behaviour, then $c_{T}(s)=r(T(s))\left(s \in \mathbb{R}_{+}\right)$.

Proof. We know from Lemma 12 that $r(T(s)) \geq c_{T}(s)>0\left(s \in \mathbb{R}_{+}\right)$, and so $T: \mathbb{R}_{+} \rightarrow \mathcal{L}(\mathcal{X})$ is not quasinilpotent. Hence Lemma 15 implies that $r(T(\cdot))$ is a character of $\mathbb{R}_{+}$. Now Corollary 10 yields the assertion.

However, the following example shows that the spectral radius function and the limit functional can be different.

EXAMPLE 17. On the semigroup $\mathbb{R}_{+}^{2}$, consider the weight function $w(x, y)$ $:=e^{x(1-y)}+1$ and the Banach space $C_{w}$ of continuous functions $f: \mathbb{R}_{+}^{2} \rightarrow \mathbb{C}$ satisfying $f \mid \partial \mathbb{R}_{+}^{2} \equiv 0$ and $\|f\|_{w}:=\sup _{\mathbb{R}_{+}^{2}}|f(x, y)| w(x, y)<\infty$. The strongly continuous representation $T: \mathbb{R}_{+}^{2} \rightarrow \mathcal{L}\left(C_{w}\right)$ is defined by

$$
(T(s, t) f)(x, y):= \begin{cases}f(x-s, y-t) & \text { if }(x-s, y-t) \in \mathbb{R}_{+}^{2}, \\ 0 & \text { otherwise. }\end{cases}
$$

Since $\lim _{(x, y) \rightarrow \infty} w(x, y)=1$ it follows that, for every $(s, t) \in \mathbb{R}_{+}^{2}$,

$$
\lim _{(x, y) \rightarrow \infty} w_{(s, t)}(x, y) / w(x, y)=1,
$$

hence $w$ is a gauge function (satisfying $(\mathrm{H})$ ) with $c_{w} \equiv 1$. Since $w$ is submultiplicative, we have $\|T(s, t)\| \leq w(s, t)\left((s, t) \in \mathbb{R}_{+}^{2}\right)$. Taking a sequence $\left\{f_{n}\right\}_{n}$ in $C_{w}$ satisfying $0 \leq f_{n}(x, y) \leq f_{n}(1 / n, 1 / n)=1,(x, y) \in \mathbb{R}_{+}^{2}$, where the support of $f_{n}$ is included in the disc centered at $(1 / n, 1 / n)$ and of radius $1 /(2 n)$, we find that $\lim _{n}\left\|f_{n}\right\|_{w}=2$ and $\lim _{n}\left\|T(s, t) f_{n}\right\|_{w}=w(s, t)$, whence $\frac{1}{2} w(s, t) \leq\|T(s, t)\|\left((s, t) \in \mathbb{R}_{+}^{2}\right)$. This implies that $T(s, t)$ has a regular norm-function with respect to $w$. By the spectral radius formula, $r(T(s, 0))=e^{s}$.

4. The peripheral spectrum. Let $S^{\sharp}, S^{*}$ denote the characters and bounded characters of $S$, respectively. For any bounded character $\chi$ we have $|\chi(s)| \leq 1$ for every $s \in S$. We recall that $C_{\mathrm{c}}(S)$ stands for the set of continuous functions with compact support in $S$. The Fourier transform of a function $f \in C_{\mathrm{c}}(S)$ with respect to the representation $\varrho: S \rightarrow \mathcal{L}(\mathcal{X})$ is given by

$$
\widehat{f}(\varrho):=\int_{S} f(s) \varrho(s) d \mu(s) .
$$

The integral exists pointwise: $\widehat{f}(\varrho) x=\int_{S} f(s) \varrho(s) x d \mu(s)(x \in \mathcal{X})$ in the Bochner sense (see e.g. [H, Chapter 7.5]). Since the representation $\varrho$ is strongly continuous, the Uniform Boundedness Principle implies that $\|\varrho(\cdot)\|$ is bounded on the support of $f$, hence $\widehat{f}(\varrho) \in \mathcal{L}(\mathcal{X})$. The characters of $S$ 
are one-dimensional representations, hence the formula for $\widehat{f}(\chi)$ also makes sense when $\chi \in S^{\sharp}$.

Since $S$ is a locally compact Hausdorff space it is easy to see by the Urysohn lemma that for each $\chi \in S^{\sharp}$ there exists an $f \in C_{\mathrm{c}}(S)$ such that $\widehat{f}(\chi) \neq 0$. Moreover, for all distinct $\chi_{1}, \chi_{2} \in S^{\sharp}$ we can find an $f \in C_{\mathrm{c}}(S)$ such that $\widehat{f}\left(\chi_{1}\right) \neq \widehat{f}\left(\chi_{2}\right)$. In other words, the functions $\widehat{f}$ separate the points of $S^{\sharp}$ from each other and from zero.

We shall define the spectrum for unbounded representations related to Lyubich's $\delta$-spectrum [L] and the algebraic and balanced spectra of [K2]. In the discrete case the algebraic spectrum of the representation $\varrho$ was defined in $[\mathrm{K} 2]$ in the following way. Let $\mathcal{A}_{\varrho}$ denote the Banach algebra generated by the set $\{\varrho(s): s \in S\}$. Then $\sigma_{\mathrm{a}}(\varrho):=\left\{h \circ \varrho: h \in \Sigma\left(\mathcal{A}_{\varrho}\right)\right\}$ where $\Sigma\left(\mathcal{A}_{\varrho}\right)$ is the Gelfand spectrum of the commutative Banach algebra $\mathcal{A}_{\varrho}$. It is easy to see that $\sigma_{\mathrm{a}}(\varrho)$ is equal to the set of those characters $\chi \in S^{\sharp}$ satisfying $\left|\sum_{s \in \mathcal{F}} a_{s} \chi(s)\right| \leq\left\|\sum_{s \in \mathcal{F}} a_{s} \varrho(s)\right\|$ for all finite subsets $\mathcal{F}$ of $S$ and $a_{s} \in \mathbb{C}$. In the continuous case one is led to the following extensions of the concept of algebraic and balanced spectra.

Definition. The algebraic spectrum of the representation $\varrho$ is

$$
\sigma_{\mathrm{a}}(\varrho):=\left\{\chi \in S^{\sharp}:|\widehat{f}(\chi)| \leq\|\widehat{f}(\varrho)\| \text { for all } f \in C_{\mathrm{c}}(S)\right\} .
$$

The balanced spectrum is

$$
\sigma_{\mathrm{b}}(\varrho):=\sigma_{\mathrm{a}}(\varrho) \cap S_{\mathrm{b}}^{\sharp},
$$

where $S_{\mathrm{b}}^{\sharp}:=\left\{\chi \in S^{\sharp}: \chi(s) \neq 0\right.$ for all $\left.s \in S\right\}$.

The spectrum of $\varrho$ with regular norm-function is

$$
\sigma(\varrho):=\left\{\chi \in \sigma_{\mathrm{a}}(\varrho):|\chi| \leq c_{\varrho}\right\},
$$

where $c_{\varrho}$ denotes the limit functional of $\varrho$.

We are going to show that the balanced spectrum $\sigma_{\mathrm{b}}(\varrho)$ is contained in the spectrum $\sigma(\varrho)$. We need the following lemma.

Lemma 18. If the norm-function of the representation $\varrho: S \rightarrow \mathcal{L}(\mathcal{X})$ is continuous at $s_{0} \in S$, then $\left|\chi\left(s_{0}\right)\right| \leq\left\|\varrho\left(s_{0}\right)\right\|$ for every $\chi \in \sigma_{\mathrm{a}}(\varrho)$.

Proof. Fix a character $\chi \in \sigma_{\mathrm{a}}(\varrho)$ and $\varepsilon>0$. Since the functions $\|\varrho(\cdot)\|$ and $\chi$ are continuous at $s_{0}$, there exists an open set $V \subseteq S$ containing $s_{0}$ such that $\left|\|\varrho(s)\|-\left\|\varrho\left(s_{0}\right)\right\|\right|<\varepsilon$ and $\left|\chi(s)-\chi\left(s_{0}\right)\right|<\varepsilon$ for every $s \in V$. By the Urysohn lemma we can find a nonnegative function $f_{0} \in C_{\mathrm{c}}(S)$ with support in $V$ and $\int_{S} f_{0} d \mu=1$. Then

$$
\begin{aligned}
\left\|\widehat{f}_{0}(\varrho) x\right\| & \leq \int_{V} f_{0}(s)\|\varrho(s) x\| d \mu(s) \leq \int_{V} f_{0}(s)\left(\left\|\varrho\left(s_{0}\right)\right\|+\varepsilon\right)\|x\| d \mu(s) \\
& =\left(\left\|\varrho\left(s_{0}\right)\right\|+\varepsilon\right)\|x\| \quad(x \in \mathcal{X})
\end{aligned}
$$


and so $\left\|\widehat{f_{0}}(\varrho)\right\| \leq\left\|\varrho\left(s_{0}\right)\right\|+\varepsilon$. As also $\left|\widehat{f_{0}}(\chi)-\chi\left(s_{0}\right)\right|<\varepsilon$, we infer that

$$
\left|\chi\left(s_{0}\right)\right|-\varepsilon<\left|\widehat{f}_{0}(\chi)\right| \leq\left\|\widehat{f}_{0}(\varrho)\right\| \leq\left\|\varrho\left(s_{0}\right)\right\|+\varepsilon .
$$

Since $\varepsilon$ can be arbitrarily small, we conclude that $\left|\chi\left(s_{0}\right)\right| \leq\left\|\varrho\left(s_{0}\right)\right\|$.

Proposition 19. If $\varrho: S \rightarrow \mathcal{L}(\mathcal{X})$ is a representation with regular norm-function, then $\sigma_{\mathrm{b}}(\varrho) \subseteq \sigma(\varrho)$.

Proof. Let $S_{\mathrm{c}}(\varrho)$ stand for the set of points $s \in S$ where $\|\varrho(\cdot)\|$ is continuous. The norm-function $\|\varrho(\cdot)\|$, being the least upper bound of the continuous functions $\|\varrho(\cdot) x\|(x \in \mathcal{X},\|x\|=1)$, is lower semicontinuous. It follows that $S \backslash S_{\mathrm{c}}(\varrho)$ is of first Baire category, and so $S_{\mathrm{c}}(\varrho)$ is dense in $S$. (See, e.g., [E, p. 87].)

Fix $\chi \in \sigma_{\mathrm{b}}(\varrho)$. We know from Lemma 18 that $|\chi(s)| \leq\|\varrho(s)\|$ for every $s \in S_{\mathrm{c}}(\varrho)$. Consider the representation $\widetilde{\varrho}:=\chi^{-1} \varrho$. It is clear that $\|\widetilde{\varrho}(s)\| \geq 1$ for every $s \in S_{\mathrm{c}}(\varrho)$. Assume that $\left\|\widetilde{\varrho}\left(s_{0}\right)\right\|<1$ for some $s_{0} \in S$. Choose a nonempty open set $V \subset S^{\circ}$ with compact closure. Then $\alpha:=\sup \{\|\varrho(s)\|$ : $s \in V\}$ is finite. For any $n \in \mathbb{N}$ and $s \in V$, we have $\left\|\widetilde{\varrho}\left(n s_{0}+s\right)\right\| \leq\left\|\widetilde{\varrho}\left(s_{0}\right)\right\|^{n} \alpha$. If $n_{0} \in \mathbb{N}$ is sufficiently large, then $\left\|\widetilde{\varrho}\left(s_{0}\right)\right\|^{n_{0}} \alpha<1$, and so $\left\|\widetilde{\varrho}\left(n_{0} s_{0}+s\right)\right\|<1$ for every $s \in V$. However, $\left(n_{0} s_{0}+V\right) \cap S_{\mathrm{c}}(\varrho)$ being non-empty, we arrive at a contradiction. Thus $\|\widetilde{\varrho}(s)\| \geq 1$ and so $|\chi(s)| \leq\|\varrho(s)\|$ for all $s \in S$.

Assume $\varrho$ has $p$-regular norm-function. Since $|\chi(s)| \leq\|\varrho(s)\| \leq p(s)$ $(s \in S)$, we infer that $\widetilde{p}(s) \geq 1(s \in S)$ for the gauge function $\widetilde{p}=|\chi|^{-1} p$. Lemma 9 yields $c_{\widetilde{p}} \geq 1$, and the relations $c_{\widetilde{p}}=|\chi|^{-1} c_{p}=|\chi|^{-1} c_{\varrho}$ imply $|\chi| \leq c_{\varrho}$, and so $\chi \in \sigma(\varrho)$.

REMARKS. (a) For any $\chi \in \sigma_{\mathrm{b}}(\varrho)$ we have seen that $|\chi(s)|^{n}=|\chi(n s)| \leq$ $\|\varrho(n s)\| \leq\left\|\varrho(s)^{n}\right\|(s \in S, n \in \mathbb{N})$, whence $|\chi(s)| \leq r(\varrho(s))$, for an arbitrary representation $\varrho$.

(b) If $S=\mathbb{R}_{+}^{n}$, then it can be easily checked that every character $\chi \in$ $\left(\mathbb{R}_{+}^{n}\right)^{\#}$ is non-vanishing, thus $\sigma_{\mathrm{a}}(\varrho), \sigma_{\mathrm{b}}(\varrho)$ and $\sigma(\varrho)$ coincide in that case. (Indeed, let $\left\{e_{i}\right\}_{i=1}^{n}$ be the standard basis in $\mathbb{R}_{+}^{n}$. If $\chi(t)=0$ for some $t=\left(t_{1}, \ldots, t_{n}\right) \in \mathbb{R}_{+}^{n}$, then the equality $\chi(t)=\prod_{i=1}^{n} \chi\left(e_{i}\right)^{t_{i}}$ implies that $\chi\left(e_{j}\right)=0$ for some $1 \leq j \leq n$. Hence $\chi$ vanishes on a dense set. Since $\chi(0)=1$ it follows that $\chi$ is not continuous.)

The most important spectrum that we need is the peripheral spectrum.

Definition. The peripheral spectrum of the representation $\varrho: S \rightarrow \mathcal{L}(\mathcal{X})$ with regular norm-function is defined by

$$
\sigma_{\text {per }}(\varrho):=\left\{\chi \in \sigma(\varrho):|\chi(s)|=c_{\varrho}(s) \text { for all } s \in S\right\} .
$$

Finally, we define the point spectrum.

Definition. The point spectrum of the representation $\varrho: S \rightarrow \mathcal{L}(\mathcal{X})$ is $\sigma_{\mathrm{p}}(\varrho):=\left\{\chi \in S^{\sharp}:\right.$ there exists $0 \neq x \in \mathcal{X}$ with $\varrho(s) x=\chi(s) x$ for all $\left.s \in S\right\}$. 
The adjoint $\varrho^{*}(s):=\varrho(s)^{*}(s \in S)$ of $\varrho$ is not necessarily strongly continuous, hence the spectrum of $\varrho^{*}$ cannot be defined in general. However, there is no difficulty in defining $\sigma_{\mathrm{p}}\left(\varrho^{*}\right)$ analogously to $\sigma_{\mathrm{p}}(\varrho)$.

Multiplication of the representation $\varrho$ (with regular norm-function) by a nonvanishing character $\tau$ yields a representation (with regular normfunction), whose different kinds of spectra can be naturally derived from the spectra of $\varrho$.

LEMMA 20. If $\tau \in S_{\mathrm{b}}^{\sharp}$ then $\sigma_{\mathrm{a}}(\tau \varrho)=\tau \sigma_{\mathrm{a}}(\varrho), \sigma_{\mathrm{b}}(\tau \varrho)=\tau \sigma_{\mathrm{b}}(\varrho), \sigma(\tau \varrho)=$ $\tau \sigma(\varrho), \sigma_{\mathrm{per}}(\tau \varrho)=\tau \sigma_{\mathrm{per}}(\varrho), \sigma_{\mathrm{p}}(\tau \varrho)=\tau \sigma_{\mathrm{p}}(\varrho)$ and $\sigma_{\mathrm{p}}\left(\tau \varrho^{*}\right)=\tau \sigma_{\mathrm{p}}\left(\varrho^{*}\right)$.

Proof. Consider the bijection $\Psi: C_{\mathrm{c}}(S) \rightarrow C_{\mathrm{c}}(S), \Psi(f):=f \tau$. Since $(\Psi(f))^{\wedge}(\chi)=\widehat{f}(\tau \chi)$ and $(\Psi(f))^{\wedge}(\varrho)=\widehat{f}(\tau \varrho)$ for every $f \in C_{\mathrm{c}}(S)$, we infer that $\sigma_{\mathrm{a}}(\tau \varrho)=\tau \sigma_{\mathrm{a}}(\varrho)$, whence $\sigma_{\mathrm{b}}(\tau \varrho)=\tau \sigma_{\mathrm{b}}(\varrho)$ readily follows. In view of $c_{\tau \varrho}=\tau c_{\varrho}$ we deduce that $\sigma(\tau \varrho)=\tau \sigma(\varrho)$ and $\sigma_{\text {per }}(\tau \varrho)=\tau \sigma_{\text {per }}(\varrho)$. Finally, the equalities $\sigma_{\mathrm{p}}(\tau \varrho)=\tau \sigma_{\mathrm{p}}(\varrho)$ and $\sigma_{\mathrm{p}}\left(\tau \varrho^{*}\right)=\tau \sigma_{\mathrm{p}}\left(\varrho^{*}\right)$ are immediate consequences of the definition.

Let us equip $S^{\sharp}$ with the compact-open topology, so that convergence of a net in $S^{\sharp}$ means uniform convergence on compact subsets of $S$. The closed set $S^{*}$ of bounded characters can be identified with the Gelfand spectrum $\Sigma\left(L^{1}(S)\right)$ of the abelian Banach algebra $L^{1}(S)$. Indeed, $L^{1}(S)$ with the multiplication $(f * g)(s)=\int_{S} f(s-t) g(t) d \mu(t)$ is a closed subalgebra of the abelian Banach algebra $L^{1}(G)$. Adaptation of the proof of [R, Theorem 1.2.2] to the semigroup setting shows that the mapping

$$
\Lambda: S^{*} \rightarrow \Sigma\left(L^{1}(S)\right), \quad \chi \mapsto h_{\chi}, \quad \text { where } \quad h_{\chi}(f)=\int_{S} f \chi=\widehat{f}(\chi),
$$

is a bijection. We recall that $\Sigma\left(L^{1}(S)\right)$ is a locally compact Hausdorff space with the Gelfand topology, induced by the weak-* topology of the dual space of $L^{1}(S)$. Notice also that if $\check{f} \in C_{0}\left(\Sigma\left(L^{1}(S)\right)\right)$ is the Gelfand transform of $f \in L^{1}(S)$, then $\check{f}\left(h_{\chi}\right)=h_{\chi}(f)=\widehat{f}(\chi)$ for every $\chi \in S^{*}$.

Proposition 21. The mapping $\Lambda: S^{*} \rightarrow \Sigma\left(L^{1}(S)\right), \chi \mapsto h_{\chi}$, is a homeomorphism, and so $S^{*}$ is locally compact in the compact-open topology.

Proof. For the sake of completeness we sketch the proof. The continuity of $\Lambda$ is an immediate consequence of the facts that $C_{\mathrm{c}}(S)$ is dense in $L^{1}(S)$ and that $\|h\|=1$ for every $h \in \Sigma\left(L^{1}(S)\right)$.

Assume now that a net $\left\{h_{\nu}=h_{\chi_{\nu}}\right\}_{\nu \in \mathcal{N}}$ converges to $h=h_{\chi}$, that is, $\lim _{\nu} h_{\nu}(g)=h(g)$ for every $g \in L^{1}(S)$. Fix $f \in L^{1}(S)$ so that $h(f) \neq 0$, and consider the equalities

$$
h_{\nu}(f) \chi_{\nu}(t)=h_{\nu}\left(f_{-t}\right) \quad(\nu \in \mathcal{N}, t \in S)
$$


and

$$
h(f) \chi(t)=h\left(f_{-t}\right) \quad(t \in S) .
$$

(See the proof of [R, Theorem 1.2.2].) Since the mapping $\varphi: S \rightarrow L^{1}(S), t \mapsto$ $f_{-t}$, is continuous, we can easily deduce that $\left\{\chi_{\nu}\right\}_{\nu}$ converges to $\chi$ uniformly on compact sets.

For any $\tau \in S_{\mathrm{b}}^{\sharp}$, the multiplication $M_{\tau}: S^{\sharp} \rightarrow S^{\sharp}, \chi \mapsto \tau \chi$, is clearly a homeomorphism, and so $\tau S^{*}$ is also a locally compact Hausdorff space with the compact-open topology.

Now we turn to the spectrum of a representation.

Proposition 22. Let $\varrho: S \rightarrow \mathcal{L}(\mathcal{X})$ be a representation of regular normbehaviour. The spectrum $\sigma(\varrho)$ of $\varrho$ is a locally compact Hausdorff space with the compact-open topology, as also is its closed subset $\sigma_{\text {per }}(\varrho)$.

Proof. It is easy to see that $\sigma_{\mathrm{a}}(\varrho)$ is closed in $S^{\sharp}$. Since the limit functional $c_{\varrho}$ belongs to $S_{\mathrm{b}}^{\sharp}$, it follows from Proposition 21 that $c_{\varrho} S^{*}$ is locally compact. Then the closed subset $\sigma(\varrho)=\sigma_{\mathrm{a}}(\varrho) \cap c_{\varrho} S^{*}$ of $c_{\varrho} S^{*}$ is also locally compact.

Remarks. (a) Assume that the representation $\varrho: S \rightarrow \mathcal{L}(\mathcal{X})$ is bounded: $\alpha:=\sup \{\|\varrho(s)\|: s \in S\}<\infty$. If $\left\|\varrho\left(s_{0}\right)\right\|<1$ for some $s_{0} \in S$, then the inequalities $\left\|\varrho\left(n s_{0}+s\right)\right\| \leq\left\|\varrho\left(s_{0}\right)\right\|^{n} \alpha(n \in \mathbb{N})$ show that $\lim _{s}\|\varrho(s)\|=0$, i.e., $\varrho$ is uniformly stable.

Assuming that $\|\varrho(s)\| \geq 1$ for every $s \in S$, we can see that $\varrho$ is of regular norm-behaviour with respect to the gauge function $p(s):=\alpha(s \in S)$. The limit functional $c_{\varrho}$ of $\varrho$ is clearly the constant 1 function. Thus $\sigma_{\text {per }}(\varrho)$ coincides with the unitary spectrum $\sigma_{\mathrm{u}}(\varrho):=\{\chi \in \sigma(\varrho):|\chi|=1\}$ of $\varrho$. It can be easily verified that also $\sigma_{\mathrm{a}}(\varrho)=\sigma(\varrho)$. Indeed, if $\left|\chi\left(s_{0}\right)\right|>1$ for some $\chi \in S^{\sharp}$ and $s_{0} \in S$, then $\lim _{n}\left|\chi\left(n s_{0}\right)\right|=\infty$, and so the Urysohn lemma yields $\sup \left\{|\widehat{f}(\chi)|: 0 \leq f \in C_{\mathrm{c}}(S),\|f\|_{1}=1\right\}=\infty$. Since $\sup \{\|\widehat{f}(\varrho)\|: 0 \leq$ $\left.f \in C_{\mathrm{c}}(S),\|f\|_{1}=1\right\} \leq \alpha$, we see that $\chi$ cannot belong to $\sigma_{\mathrm{a}}(\varrho)$.

The Fourier transforms $\widehat{f}(\chi)$ and $\widehat{f}(\varrho)$ are clearly defined for every $f \in$ $L^{1}(S)$ and $\chi \in S^{*}$. As $C_{\mathrm{c}}(S)$ is dense in $L^{1}(S)$, we conclude that if the inequality $|\widehat{f}(\chi)| \leq\|\widehat{f}(\varrho)\|$ holds for every $f \in C_{\mathrm{c}}(S)$ then it also does for every $f \in L^{1}(S)$. Thus $\sigma(\varrho)$ coincides with the spectrum introduced by Batty and Vũ for bounded representations in [BV]. We also recall that this concept is an adaptation of the finite $L$-spectrum and the Arveson spectrum, defined for group representations, to the semigroup setting (see $[\mathrm{L}]$ and $[\mathrm{D}]$ ).

(b) Let $\varrho: S \rightarrow \mathcal{L}(\mathcal{X})$ be a representation of regular norm-behaviour. Since $c_{\varrho} \in S_{\mathrm{b}}^{\sharp}$, the representation $\widetilde{\varrho}:=c_{\varrho}^{-1} \varrho: S \rightarrow \mathcal{L}(\mathcal{X})$ is also of regular norm-behaviour and $c_{\widetilde{\varrho}}=1$. Clearly $|\chi|=1$ for $\chi \in \sigma_{\text {per }}(\widetilde{\varrho})$. Hence every $\chi \in \sigma_{\text {per }}(\widetilde{\varrho})$ can be uniquely extended to a character $\widetilde{\chi}$ of the extension 
group $G$. We conclude that $\sigma_{\text {per }}(\widetilde{\varrho})$ can be identified with the unitary spectrum $\operatorname{Sp}_{\mathrm{u}}(\widetilde{\varrho})$ introduced in $[\mathrm{BY}]$, namely $\sigma_{\mathrm{per}}(\widetilde{\varrho})=\left\{\widetilde{\chi} \mid S: \widetilde{\chi} \in \operatorname{Sp}_{\mathrm{u}}(\widetilde{\varrho})\right\}$. Therefore $\sigma_{\text {per }}(\varrho)=\left\{c_{\varrho}(\widetilde{\chi} \mid S): \widetilde{\chi} \in \operatorname{Sp}_{\mathrm{u}}(\widetilde{\varrho})\right\}$ by Lemma 20 .

5. Associated isometric representation. The key ingredient in proving the stability of $\varrho$ is the transmission of the spectral conditions to a related isometric representation. Such an associated representation is discussed in the following theorem.

TheOREM 23. For any representation $\varrho: S \rightarrow \mathcal{L}(\mathcal{X})$ with $p$-regular normfunction, there exist an isometric representation $\psi: S \rightarrow \mathcal{L}(\mathcal{Y})$ on a Banach space $\mathcal{Y}$ and a contractive transformation $Q \in \mathcal{L}(\mathcal{X}, \mathcal{Y})$ such that:

(i) $\operatorname{ker} Q=\left\{x \in \mathcal{X}: \operatorname{a-lim}_{s}\|\varrho(s) x\| / p(s)=0\right\}$, and $\operatorname{ran} Q$ is dense in $\mathcal{Y}$,

(ii) $Q \varrho(s)=c_{\varrho}(s) \psi(s) Q$ for every $s \in S$,

(iii) for every operator $C$ in the commutant $\{\varrho(S)\}^{\prime}$, there exists a unique operator $D \in\{\psi(S)\}^{\prime}$ such that $Q C=D Q$; furthermore, the mapping $\gamma:\{\varrho(S)\}^{\prime} \rightarrow\{\psi(S)\}^{\prime}, C \mapsto D$, is a contractive algebra-homomorphism,

(iv) $\sigma(\varrho) \supseteq c_{\varrho} \sigma(\psi), \sigma_{\mathrm{per}}(\varrho) \supseteq c_{\varrho} \sigma_{\mathrm{per}}(\psi), \sigma_{\mathrm{p}}\left(\varrho^{*}\right) \supseteq c_{\varrho} \sigma_{\mathrm{p}}\left(\psi^{*}\right)$.

Proof. (i) Define a seminorm on $\mathcal{X}$ by

$$
\ell(x):=\sup \{m(\|\varrho(\cdot) x\| / p): m \in M(S)\} .
$$

Clearly $\ell(x) \leq\|x\|$, and $\ell(x)=0$ if and only if a-lim $\left\|_{s}\right\|(s) x \| / p(s)=0$. Let $\mathcal{Y}$ be the completion of $\mathcal{X} / \operatorname{ker} \ell$ with the norm $\|x+\operatorname{ker} \ell\|:=\ell(x)$, and let $Q: \mathcal{X} \rightarrow \mathcal{Y}$ denote the natural embedding. Obviously, $\operatorname{ran} Q$ is dense.

(ii) In view of Lemma 8 , for any $x \in \mathcal{X}$ and $t \in S$ we have

$$
\begin{aligned}
\|Q \varrho(t) x\|=\ell(\varrho(t) x) & =\sup _{m \in M(S)} m\left(\frac{\|\varrho(\cdot+t) x\|}{p}\right) \\
& =\sup _{m \in M(S)} m\left(\frac{\|\varrho(\cdot+t) x\|}{p_{t}} \frac{p_{t}}{p}\right) \\
& =c_{\varrho}(t) \sup _{m \in M(S)} m\left(\frac{\|\varrho(\cdot) x\|}{p}\right) \\
& =c_{\varrho}(t) \ell(x)=c_{\varrho}(t)\|Q x\| .
\end{aligned}
$$

Hence there exists a unique isometry $\psi(t) \in \mathcal{L}(\mathcal{Y})$ such that $Q \varrho(t)=$ $c_{\varrho}(t) \psi(t) Q$. This intertwining relation readily implies that $\psi(s+t)=$ $\psi(s) \psi(t)$ so $\psi$ is a homomorphism.

The inequality

$$
\begin{aligned}
\left\|c_{\varrho}(s) \psi(s) Q x-c_{\varrho}(t) \psi(t) Q x\right\| & =\|Q \varrho(s) x-Q \varrho(t) x\| \\
& \leq\|Q\|\|\varrho(s) x-\varrho(t) x\| \quad(x \in \mathcal{X})
\end{aligned}
$$


shows that the function $c_{\varrho}(t) \psi(t) y$ is continuous in $t$ for every $y$ in the dense range of $Q$, and hence so is $\psi(t) y$. As $\psi$ is isometric, it is strongly continuous. Therefore $\psi$ is an isometric representation.

(iii) For any $C \in\{\varrho(S)\}^{\prime}$, we have

$$
\|Q C x\|=\sup _{m \in M(S)} m\left(\frac{\|C \varrho(\cdot) x\|}{p}\right) \leq\|C\| \ell(x)=\|C\|\|Q x\|,
$$

so there exists a unique $D \in \mathcal{L}(\mathcal{Y})$ such that $Q C=D Q$. Clearly $\|D\| \leq$ $\|C\|$, and it is easy to check that $D \in\{\psi(S)\}^{\prime}$ and that $\gamma$ is an algebrahomomorphism.

(iv) Since $\widehat{\hat{f}}(\varrho) \in\{\varrho(S)\}^{\prime}$ and $Q \widehat{f}(\varrho)=\widehat{f}\left(c_{\varrho} \psi\right) Q$, we have $\gamma(\widehat{f}(\varrho))=$ $\widehat{f}\left(c_{\varrho} \psi\right)\left(f \in C_{\mathrm{c}}(S)\right)$. As $\gamma:\{\varrho(S)\}^{\prime} \rightarrow\{\psi(S)\}^{\prime}$ is contractive by (iii), we infer that $\left\|\widehat{f}\left(c_{\varrho} \psi\right)\right\| \leq\|\widehat{f}(\varrho)\|$, whence $c_{\varrho} \sigma_{\mathrm{a}}(\psi)=\sigma_{\mathrm{a}}\left(c_{\varrho} \psi\right) \subseteq \sigma_{\mathrm{a}}(\varrho)$ (see Lemma $20)$. Since the limit functional of the isometric representation $\psi$ is clearly the constant 1 function, we conclude that $c_{\varrho} \sigma(\psi) \subseteq \sigma(\varrho)$ and $c_{\varrho} \sigma_{\text {per }}(\psi) \subseteq$ $\sigma_{\text {per }}(\varrho)$. Finally, the equalities $\operatorname{ker} Q^{*}=\{0\}$ and $\varrho^{*}(s) Q^{*}=c_{\varrho}(s) Q^{*} \psi^{*}(s)$ $(s \in S)$ readily imply that $\sigma_{\mathrm{p}}\left(\varrho^{*}\right) \supseteq c_{\varrho} \sigma_{\mathrm{p}}\left(\psi^{*}\right)$.

We can easily deduce the following corollary.

Corollary 24. If $\varrho: S \rightarrow \mathcal{L}(\mathcal{X})$ is a representation with p-regular

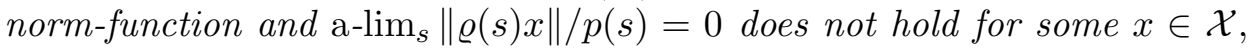
then $\sigma_{\text {per }}(\varrho)$ is not empty.

Proof. The conditions ensure that the associated isometric representation $\psi$ of Theorem 23 acts on a non-zero Banach space $\mathcal{Y}$. Thus $\sigma_{\text {per }}(\psi)$ is non-empty by $\left[\mathrm{BV}\right.$, Corollary 3.3]. Since $c_{\varrho} \sigma_{\text {per }}(\psi) \subset \sigma_{\text {per }}(\varrho)$, we infer that $\sigma_{\text {per }}(\varrho)$ is not empty.

6. The stability theorem. Now, we prove a stability result in terms of almost convergence, concerning representations with regular norm-function:

Theorem 25. Let $\varrho: S \rightarrow \mathcal{L}(\mathcal{X})$ be a representation with a p-regular norm-function. If $\sigma_{\mathrm{per}}(\varrho)$ is countable and $\sigma_{\mathrm{p}}\left(\varrho^{*}\right) \cap\left\{\chi:|\chi|=c_{\varrho}\right\}=\emptyset$ then

$$
\operatorname{a-lim}_{s} \frac{\|\varrho(s) x\|}{p(s)}=0 \quad \text { for all } x \in \mathcal{X} .
$$

Proof. If the conclusion fails to hold then by Theorem 23 the associated isometric representation $\psi: S \rightarrow \mathcal{L}(\mathcal{Y})$ acts on a non-zero Banach space $\mathcal{Y}$. Hence by [BV, Corollary 3.3], $\sigma_{\text {per }}(\psi)$ is not empty. The relation $\sigma_{\text {per }}(\varrho) \supseteq$ $c_{\varrho} \sigma_{\text {per }}(\psi)$ implies that $\sigma_{\text {per }}(\psi)$ is also countable. Thus $\sigma_{\text {per }}(\psi)$ contains an eigenvalue $\chi$ of $\psi^{*}$ by [BV, Proposition 4.1]. Applying Theorem 23 again, we conclude that $c_{\varrho} \chi \in \sigma_{\mathrm{p}}\left(\varrho^{*}\right) \cap\left\{\chi:|\chi|=c_{\varrho}\right\}$, which is a contradiction.

Applying Proposition 7 we obtain the following corollary. 
Corollary 26. Let $\varrho: S \rightarrow \mathcal{L}(\mathcal{X})$ be a representation with a p-regular norm-function. If $\sigma_{\mathrm{per}}(\varrho)$ is countable and $\sigma_{\mathrm{p}}\left(\varrho^{*}\right) \cap\left\{\chi:|\chi|=c_{\varrho}\right\}=\emptyset$ then

$$
\lim _{n \rightarrow \infty} \frac{1}{\mu\left(K_{n}\right)} \int_{K_{n}} \frac{\|\varrho(s) x\|}{p(s)} d \mu(s)=0 \quad \text { for all } x \in \mathcal{X},
$$

where $\left\{K_{n}\right\}_{n}$ is any Følner sequence.

In view of Proposition 11 and Remark (a) at the end of Section 4, Theorem 25 is a generalization of the stability result [BV, Theorem 4.2] concerning bounded representations. In view of Remark (b) at the end of Section 4, the spectral conditions of Theorem 25 are essentially the same as those in the main result Theorem 3.2 of [BY]. The differences lie in the norm-condition on $\varrho$ and in the nature of convergence expressing stability.

Acknowledgements. The authors are indebted to the referee for the valuable suggestions which made the presentation more streamlined and transparent.

\section{References}

[BP] B. Basit and A. J. Pryde, Ergodicity and stability of orbits of unbounded semigroup representations, J. Austral. Math. Soc. 77 (2004), 209-232.

[B] C. J. K. Batty, Asymptotic behaviour of semigroups of operators, in: Banach Center Publ. 30, Inst. Math., Polish Acad. Sci., Warszawa, 1994, 35-52.

[BV] C. J. K. Batty and Q. P. Vũ, Stability of strongly continuous representations of abelian semigroups, Math. Z. 209 (1992), 75-88.

[BY] C. J. K. Batty and S. B. Yeates, Weighted and local stability of semigroups of operators, Math. Proc. Cambridge Philos. Soc. 129 (2000), 85-98.

[C] J. B. Conway, A Course in Functional Analysis, 2nd ed., Springer, New York, 1990.

[D] E. B. Davies, One-Parameter Semigroups, Academic Press, London, 1980.

[E] R. Engelking, General Topology, 2nd ed., Heldermann, Berlin, 1989.

[EN] K.-J. Engel and R. Nagel, One-Parameter Semigroups for Linear Evolution Equations, Springer, New York, 1999.

[HR] E. Hewitt and K. A. Ross, Abstract Harmonic Analysis, Vol. 1, Springer, Berlin, 1963.

[H] E. Hille, Methods in Classical and Functional Analysis, Addison-Wesley, Reading, MA, 1972.

[K] J. Kelley, General Topology, Van Nostrand, New York, 1969.

[K1] L. Kérchy, Operators with regular norm-sequences, Acta Sci. Math. (Szeged) 63 (1997), 571-605.

[K2] - Representations with regular norm-behaviour of discrete abelian semigroups, ibid. 65 (1999), 701-726.

[Lo] G. G. Lorentz, A contribution to the theory of divergent sequences, Acta Math. 80 (1948), 167-190.

[L] Y. I. Lyubich, Introduction to the Theory of Banach Representations of Groups, Birkhäuser, Basel, 1988. 
[LP] Y. I. Lyubich and Q. P. Vũ, Asymptotic stability of linear differential equations on Banach spaces, Studia Math. 88 (1988), 37-42.

[P] A. L. T. Paterson, Amenability, Amer. Math. Soc., Providence, RI, 1988.

[Pe] G. Pedersen, Analysis Now, Springer, New York, 1988.

[R] W. Rudin, Fourier Analysis on Groups, Interscience, New York, 1962.

[V] Q. P. Vũ, Semigroups with nonquasianalytic growth, Studia Math. 104 (1993), 229-241.

Bolyai Institute

University of Szeged

Aradi Vértanúk tere 1

H-6720 Szeged, Hungary

E-mail: kerchy@math.u-szeged.hu

lzoli@math.u-szeged.hu

Received November 17, 2006

Revised version July 24, 2007 